\title{
BMJ Development of an economic model Open to assess the cost-effectiveness of hawthorn extract as an adjunct treatment for heart failure in Australia
}

\author{
Emily Ford, ${ }^{1}$ Jon Adams, ${ }^{2,3}$ Nicholas Graves ${ }^{4}$
}

To cite: Ford E, Adams J, Graves N. Development of an economic model to assess the cost-effectiveness of hawthorn extract as an adjunct treatment for heart failure in Australia. BMJ Open 2012;2:e01094.

doi:10.1136/bmjopen-2012001094

- Prepublication history and additional material for this paper are available online. To view these files please visit the journal online (http:// dx.doi.org/10.1136/bmjopen2012-001094).

Received 1 March 2012 Accepted 20 July 2012

This final article is available for use under the terms of the Creative Commons Attribution Non-Commercial 2.0 Licence; see http://bmjopen.bmj.com

For numbered affiliations see end of article

\section{Correspondence to}

Emily Ford;

emily.ford1@uqconnect.edu.au

\section{ABSTRACT}

Objective: An economic model was developed to evaluate the cost-effectiveness of hawthorn extract as an adjunctive treatment for heart failure in Australia.

Methods: A Markov model of chronic heart failure was developed to compare the costs and outcomes of standard treatment and standard treatment with hawthorn extract. Health states were defined by the New York Heart Association (NYHA) classification system and death. For any given cycle, patients could remain in the same NYHA class, experience an improvement or deterioration in NYHA class, be hospitalised or die. Model inputs were derived from the published medical literature, and the output was quality-adjusted life years (QALYs). Probabilistic sensitivity analysis was conducted. The expected value of perfect information (EVPI) and the expected value of partial perfect information (EVPPI) were conducted to establish the value of further research and the ideal target for such research.

Results: Hawthorn extract increased costs by $\$ 1866.78$ and resulted in a gain of 0.02 QALYs. The incremental cost-effectiveness ratio was $\$ 85160.33$ per QALY. The cost-effectiveness acceptability curve indicated that at a threshold of $\$ 40000$ the new treatment had a 0.29 probability of being cost-effective. The average incremental net monetary benefit (NMB) was $-\$ 1791.64$, the average NMB for the standard treatment was $\$ 92067.49$, and for hawthorn extract $\$ 90275.84$. Additional research is potentially costeffective if research is not proposed to cost more than $\$ 325$ million. Utilities form the most important target parameter group for further research.

Conclusions: Hawthorn extract is not currently considered to be cost-effective in as an adjunctive treatment for heart failure in Australia. Further research in the area of utilities is warranted.

\section{INTRODUCTION}

Heart failure is a major public health concern for all Western countries. ${ }^{1}$ In the USA and Europe, it is the most common principal diagnosis for adults admitted to

\section{ARTICLE SUMMARY}

Article focus

- The development of an economic model to evaluate the cost-effectiveness of hawthorn extract as an adjunct to standard pharmacological treatment for heart failure in Australia.

- Expected value of perfect information (EVPI) and the expected value of partial perfect information (EVPPI) analyses were conducted to examine the value of further research and the ideal target area for such research.

\section{Key messages}

- Hawthorn extract is not currently considered to be cost-effective in as an adjunctive treatment for heart failure in Australia.

- Further research is potentially cost-effective, particularly in the area of utilities.

Strengths and limitations of this study

- This is the first known attempt to examine the cost-effectiveness of hawthorn extract in addition to standard pharmacological treatment of chronic heart failure in Australia.

- There was a sparse amount of chronic heart failure data available for the Australian context.

- Another limitation was the limited availability of the effectiveness data for hawthorn extract.

hospital aged 65 years and over. In the USA around $2 \%$ of the population have heart failure (approximately 5 million people), and each year there are 500000 new cases diagnosed. $^{2}$ The estimated prevalence in Sweden is $1.5-2 \%$, approximately 135000 to 180000 people. $^{3}$

Australian data regarding the public health significance and epidemiology of heart failure are currently limited. Estimates rely on information from large-scale population studies conducted in the USA and Europe. ${ }^{1}$ It is estimated that there are approximately 300000 Australians living with chronic heart failure, and approximately 30000 new cases diagnosed 
each year, with incidence rates and prevalence rising significantly with age. ${ }^{45}$ In Australia, chronic cardiovascular diseases are associated with healthcare costs of over 5 billion dollars, and estimates put the cost of heart failure at around 1 billion dollars. ${ }^{6}$ The mortality, morbidity and healthcare costs of heart failure are therefore significant. ${ }^{4}$

Heart failure is a syndrome with a range of signs and symptoms, diagnosis is based on such signs and symptoms, including dyspnoea and fatigue, and appropriate investigations, such as echocardiogram, which confirm the presence or absence of heart failure and help determine its aetiology. ${ }^{1}$

Current treatment aims to relieve and stabilise symptoms and prolong survival by stopping, stabilising or reversing the progression of heart failure. ${ }^{7}$ There are a variety of strategies used in Australia, including nonpharmacological management, pharmacological management, lifestyle changes, and the use of supportive devices, surgery and palliative care. ${ }^{6} 8$ The pharmacological approach depends on the type of heart failure and extent of the symptoms.

Despite the availability of strategies to treat and manage the chronic disease, the disability and suffering associated with heart failure is devastating. ${ }^{7}$ Given this, and the large economic burden, it is reasonable to examine options not currently considered standard therapy. Research examining the use of complementary and alternative medicine (CAM), particularly the use of hawthorn extract, is showing promising results.

Hawthorn extract is a popular herbal medicine used worldwide, particularly for its cardiovascular properties. ${ }^{9}$ Hawthorn extract has positive inotropic, anti-inflammatory and antioxidative properties; causes peripheral and coronary vasodilation; and protects against ischaemia-induced arrhythmias. ${ }^{9}$ A recent systematic review concluded that hawthorn extract can provide significant benefits to heart failure patients as an adjunct to conventional treatment and a recent cost-effectiveness study conducted in Germany concluded that hawthorn is a cost-effective treatment option especially in the early stages of heart failure. ${ }^{10-12}$

Economic evaluation is a structured method for examining the costs and consequences involved with alternative methods of treatments and/or programmes, in order to inform which is the best alternative from a particular viewpoint. ${ }^{13}$ The goal is to improve the use of healthcare resources and improve patient care. ${ }^{14}$ When conducted rigorously, such formal analysis allows recommendation to be made with transparency regarding the methods, data sources and assumptions. ${ }^{13}$ This further allows the process to be replicated, reviewed and even challenged.

Models allow complex situations to be organised into a single coherent form that can be used to make decisions based on comprehensive consideration of the alternative interventions by capturing the essential relationships between the factors included in the model and outcomes. ${ }^{15} 16$ Markov models define diseases using clinically relevant and economically important health states, between which patients move based on the natural history of the disease, and to which cost and effectiveness outcomes are ascribed. ${ }^{16}$

There are numerous examples of cost-effectiveness modelling in heart failure that examine conventional medicine. Pharmacological, behavioural and surgical interventions have all been investigated and many found to be cost-effective. ${ }^{17}{ }^{18}$ Pharmacological agents that have cost-effectiveness evidence include ACE inhibitors (ACEIs), digoxin and $\beta$-blockers such as carvedilol and nebivolol. Multidisciplinary heart failure management, in the form of a team, usually made up of a nurse co-ordinator and support from medical staff and allied health including dieticians and physiotherapy, has also shown to be cost-effective through reductions in hopsitalisation and length of stay. ${ }^{17}{ }^{19}$ Surgical options including heart transplant, through intensive education and maximal medical therapy, have demonstrated a range of cost-effectiveness values. Cardiac resynchronisation therapy with or without an implantable cardioverterdefibrillator has shown to be cost-effective from a healthcare perspective. ${ }^{17}{ }^{20}$ Most of the recent evidence involves Markov modelling.

The increasing number of published health economic evaluations is not yet reflected in CAM. ${ }^{21-23}$ A systematic review examined whether CAM demonstrated costeffectiveness through economic evaluations. ${ }^{24}$ There was good evidence for the cost-effectiveness of several therapies in comparison with usual care, acupuncture for migraine, manual therapy for neck pain, spa therapy for Parkinson's, self-administered stress management for cancer patients undergoing chemotherapy, preoperative and postoperative oral nutritional supplementation for lower gastrointestinal tract surgery, biofeedback for patients with 'functional' disorders (eg, irritable bowel syndrome), and guided imagery, relaxation therapy and a potassium-rich diet for cardiac patients. ${ }^{24}$

There remain very few full economic evaluations today. One such evaluation examined therapeutic massage, exercise and lessons in the Alexander technique for treating persistent back pain. ${ }^{25}$ Massage, lessons in the Alexander technique and an exercise prescription all provided benefits to patients over a 12 -month period. Six lessons in the Alexander technique combined with an exercise prescription was the most cost-effective option for the National Health Service. ${ }^{25}$ Some economic evaluations of CAM have incorporated decision modelling. Recently, the costeffectiveness of adding acupuncture to usual care for chronic low-back pain was examined, using a Markov model. ${ }^{26}$ The result was an incremental cost per quality-adjusted life year (QALY) gained of KRW 3421394 , well below the threshold of South Korean Won (KRW) 20000000 . Acupuncture plus usual care was more cost-effective than usual care for these patients. The probability of collaborative treatment being cost-effective was $72.3 \%$. Expected value of perfect 
information (EVPI) analysis suggested that further research was of reasonable value. ${ }^{26}$ This highlights the need for full economic evaluations in many areas of CAM.

The aim of this study was the construction and application of an economic decision model to evaluate hawthorn treatment as an adjunct to recommended pharmacological treatment versus recommended pharmacological management for chronic heart failure in Australia. The analysis has been conducted using a health sector perspective.

\section{METHODS}

\section{Model description}

A four-state Markov model of chronic heart failure was developed based on the New York Heart Association (NYHA) classification system using Microsoft Excel (see figure 1). Classes I to IV make up four discrete health states included in the model (see table 1 for a description of the NYHA classes). A decision tree completes the model. Each NYHA class has its own decision tree. Within the decision tree patients could be hospitalised for worsening heart failure. Patients also either survived or died.

\section{Progression through the model}

A simulated cohort of 1000 patients aged 60 entered the model with NYHA class II heart failure and progressed through the model. Patients progress through the model in 1 month cycles for a duration of 5 years. After
Table 1 NYHA grading of symptoms in chronic heart failure

\begin{tabular}{ll}
\hline NYHA Class & Description \\
\hline Class I & $\begin{array}{l}\text { No symptoms and limitations in ordinary } \\
\text { physical activity. }\end{array}$ \\
Class II & $\begin{array}{l}\text { Slight limitation of physical activity. } \\
\text { Ordinary physical activity results in mild } \\
\text { symptoms such as fatigue, shortness of } \\
\text { breath and angina. }\end{array}$ \\
Class III & $\begin{array}{l}\text { Marked limitation of physical activity. Less } \\
\text { than ordinary physical activity leads to } \\
\text { symptoms. }\end{array}$ \\
& $\begin{array}{l}\text { Severely limited. Experiences symptoms } \\
\text { even at rest. }\end{array}$ \\
\hline
\end{tabular}

1 month, patients either remained in NYHA class II or improved to NYHA class I or deteriorated.

In turn, for each class of heart failure patients were either hospitalised or not hospitalised for worsening heart failure. Patients who were hospitalised or not hospitalised either survived or died. Death was a possibility from any class of heart failure. The patients accrued costs and benefits of treatment in each of the states for each cycle.

Per patient costs were required for each NYHA class. Costs were assumed to be the same for standard treatment and standard treatment with hawthorn extract, except for the additional cost of hawthorn extract. Patient health was considered as a single index utility on
Figure 1 Markov model and decision tree showing transitions between potential health states for chronic heart failure.

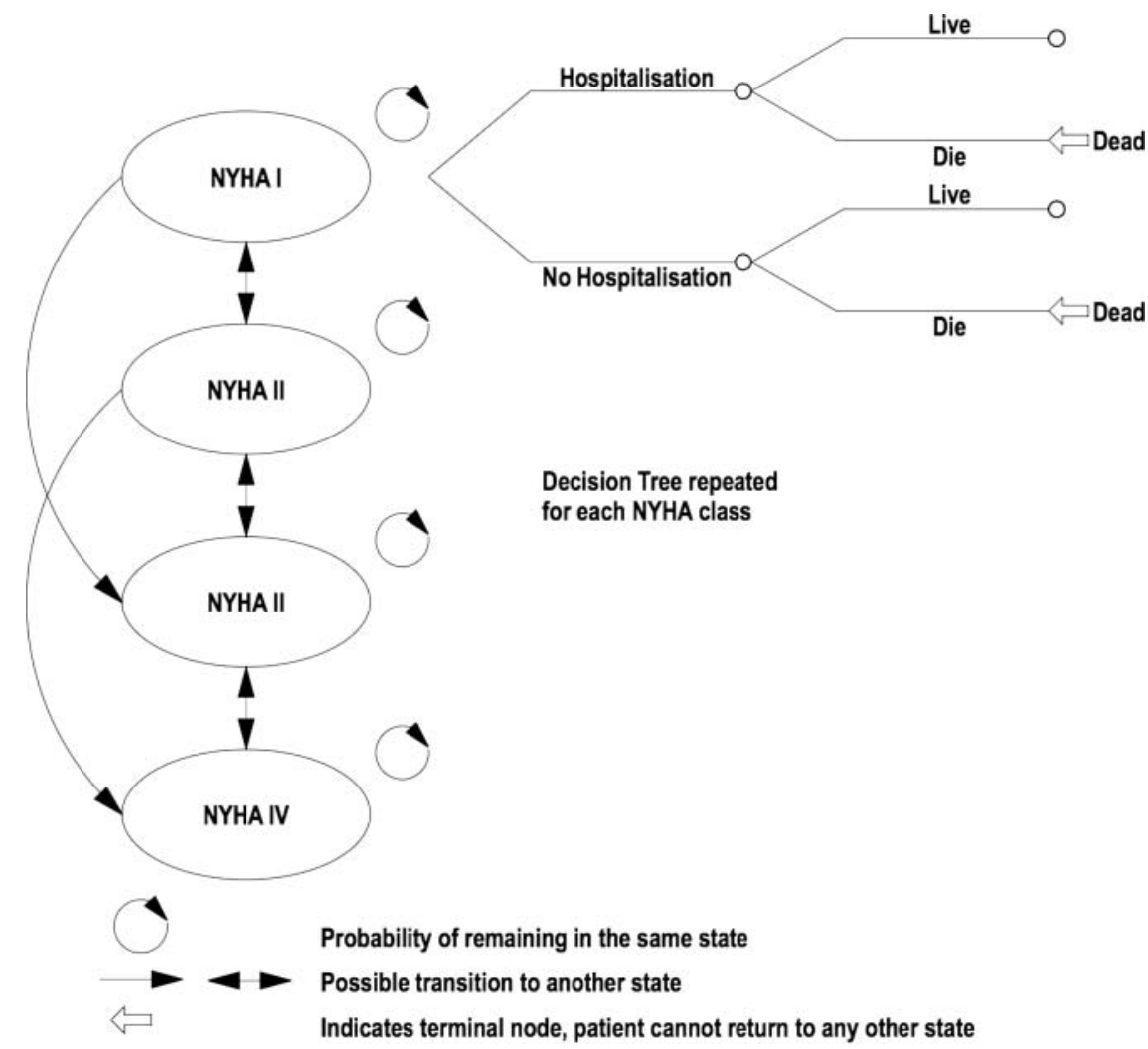


a 0 to 1 scale, where 0 represents death and 1 represents perfect health. This allows the calculation of QALYs when combined with the mortality data and the calculation of cost per QALY ratios.

Two cohorts were modelled, one receiving standard pharmacological treatment and the other receiving standard pharmacological treatment with hawthorn extract as an adjunct. The two cohorts will progress through the model in slightly different ways and as such there will be a difference in the accumulation of costs and QALYS. It is the differences in costs and QALYs that will determine the cost-effectiveness of hawthorn extract in addition to standard pharmacological treatment.

A discount rate of $3 \%$ per year was applied to the costs and benefits. This rate is a standard choice in the literature.

\section{Model construction}

Disease progression

Transition probabilities for movement between NYHA classes of heart failure were estimated from the published literature detailing the large-scale international Study of the Effects of Nebivolol Intervention on Outcomes and Re-hospitalisation in Seniors with Heart Failure (SENIORS) and personal correspondence with authors. ${ }^{18}{ }^{27}$ A thorough literature search was conducted to identify disease progression data for each NYHA class, between January 2004 and December 2009. Data were considered relevant if transition probabilities were provided for each NYHA class. The databases searched were Medline, CINAHL and the Cochrane Library. Search terms used included 'New York Heart Association', 'NYHA', 'NYHA class', 'class', 'Markov model', 'chronic heart failure' and 'heart failure'.

The search yielded a limited number of studies (17 in Medline, 3 in CINAHL and 3 in the Cochrane library), of which only the above study was considered suitable for inclusion.

Disease progression between the Markov states was assumed to be the same for standard treatment and for standard treatment with hawthorn extract, as we were unable to identify reliable data to indicate that hawthorn extract altered progression through the classes of heart failure. Transition probabilities were fixed over time. We have incorporated a difference in mortality and a difference in the hospitalisation rate between the standard treatment and the standard treatment with hawthorn extract as an adjunct, which in turn will impact on the cost and QALY outcomes.

\section{Data sources}

Mortality

Baseline mortality was derived from Australian Bureau of Statistics general population mortality data.

Mortality data were of interest if they were provided for each NYHA class and if they concerned the excess mortality from heart failure and/or cardiovascular causes. The databases searched were Medline, CINAHL and the Cochrane Library, between January 2004 and
December 2009. Search terms used included 'New York Heart Association', 'NYHA', 'NYHA class', 'class', 'Markov model', 'chronic heart failure', 'heart failure' and 'mortality'. Eighty-three papers were identified in Medline, 198 in CINAHL and 411 in Cochrane.

The death rate for cardiovascular causes was derived from the published literature detailing 1-year mortality among unselected patients with NYHA class II-IV heart failure in Switzerland. ${ }^{28}$ The death rate increased with progression from NYHA class I to NYHA class IV, and varied depending on whether the patient was hospitalised or not. A thorough search of the literature was made to identify data for each NYHA class individually, nothing was identified and the above study was the closest to ideal. Hospitalisation was considered a major factor in cost estimation, so data broken down by hospitalisation status was considered to represent the population of heart failure patients well. Also, unselected patients were considered to represent the patient cohort more accurately than studies that focused on hospitalised patients only. As data for NYHA class I were not included, an assumption was made that mortality for NYHA class I was the same as the general population mortality.

\section{Health status}

Estimates of health status were derived from the same source as the transition probabilities. ${ }^{18}{ }^{20}$ Data concerning utilities for heart failure are extremely limited, a study was identified that had specifically developed utilities for heart failure in terms of both hospitalisation and NYHA class. However, we were unable to obtain the required data despite personal correspondence with the authors. The estimated health status used was considered the next best data source.

Health status was assumed to be the same for standard treatment and standard treatment with hawthorn extract. Hospitalisation was assumed to result in a health state lower than non-hospitalisation and a -0.1 disutility was applied to hospitalisation to reflect this.

\section{Effect of hawthorn}

A literature search identified the existing research for the use of hawthorn extract in the treatment of heart failure, between January 2004 and January 2010. The search included electronic databases (Medline (472 papers) AMED (129 papers), Econlit (0 papers), CINAHL (15 papers), Cochrane Database of Systematic Reviews (71 papers)), hand searches of the literature, including hard copies of journals, and a search of the reference lists of the articles and publications found through electronic and hand searches. Personal communication with authors and experts including manufacturers and researchers in the field was also necessary to identify other sources of information and research that may not have been found using any other methods.

A wide range of search terms was used including: 'heart failure', 'chronic heart failure', 'systolic heart failure', 'congestive heart failure', 'hawthorn', 'Crataegus', 
'Crataegus oxyacantha', 'Crataegus monogyna', 'whitethorn', weissdorn', 'Crataegus laevigata', 'WS 1442', 'LI 132', 'complementary', 'alternative', 'medicine' and 'therapy'. There were several studies written in German, these were translated into English and then examined.

Publicly accessible trial registers were also searched, and the information was current up to December 2011. The Australian New Zealand Clinical Trials Registry was searched, no studies were identified. The World Health Organization International Clinical Trials Registry Platform was searched, no new relevant trials were identified. The search terms used were: 'hawthorn extract', 'hawthorn', 'crataegus', 'WS1442', 'whitehorn' and 'heart failure'.

There were no planned exclusion criteria at this stage for the patient population as any of the studies found have the potential to contribute valuable information to inform the model development.

The relative risk of mortality and relative risk of hospitalisation with hawthorn extract was derived from the Survival and Prognosis: Investigation of Crataegus Extract WS 1442 in congestive heart failure (SPICE) trial, a large-scale, international, randomised, placebocontrolled, double-blind study designed to investigate the influence of hawthorn extract on mortality of patients with congestive heart failure NYHA class II and III with at least moderately impaired left ventricular function. ${ }^{29}$ To date, there have only been two studies to examine the effect of hawthorn extract on heart failure progression in terms of mortality and hospitalisation. Most studies have focused on symptoms and exercise capability. SPICE enrolled 2681 patients, and the Hawthorn Extract Randomised Blinded Chronic Heart Failure (HERB CHF) trial enrolled 120 patients. ${ }^{30}{ }^{31}$ Meta-analysis was not considered appropriate, and therefore the data from SPICE were incorporated into the model.

\section{Costs}

No Australian data were available to estimate the hospitalisation rate and number of hospitalisations; this information was derived from a US study. ${ }^{32}$

The estimated length of stay in hospital data was obtained from Victorian Department of Health for 20102011, it was unavailable for each NYHA class, so it was assumed to be the same for all classes. ${ }^{33}$

The cost of a hospital admission per day was derived from the Queensland Government/Queensland Health Casemix Funding Model 2008-2009 Component Prices Summary ( $\$ 3775$ per day). ${ }^{34}$
Outpatient costs included general practitioner (GP) visits, pathology (urea, creatinine, electrolytes), echocardiograms and specialist visits. Estimates of the number of GP and specialist visits came from a combination of Australian sources and overseas studies due to the difficulty in finding complete Australian estimates. It was estimated that NYHA class I had 6 GP visits per year, and the remaining NYHA classes had 12 visits per year at $\$ 34.30$ per visit. Pathology was assumed to be required every 3 months at a cost of $\$ 17.80$. An echocardiogram was assumed to be performed every 2 years $(\$ 230.65)$. A specialist visit was assumed to occur twice per year (\$290 initial visit, \$194 repeat visit). If hospitalised, it was assumed patients had an extra three specialist visits and two GP visits per year. The costs came directly from the Medicare Benefits Schedule and the Queensland Government/Queensland Health Casemix Funding Model 2008-2009 Component Prices Summary. ${ }^{34} 35$

The information for which medications are taken for each NYHA class have been taken from the National Heart Foundation guidelines for the treatment of chronic heart failure in Australia. ${ }^{5}$ Information for the optimal dosages prescribed has been taken from the Australian Therapeutic Guidelines. Individual drug pricing was obtained from the most recently available online version of the Medicare Benefits Schedule. The initial version of the model has incorporated the assumption that medications are taken in $100 \%$ of patients and that dosing is optimal. The model, however, can be altered to consider different scenarios of medication prescription and consumption.

The dosage was assumed to be $900 \mathrm{mg}$ daily, consistent with the dosage used in the two most recent trials of hawthorn extract, the SPICE trial and the HERB-CHF trial. ${ }^{29} 3136$ An online search was conducted for standardised monopreparations of hawthorn leaf with flower available for purchase. Cardiomax retails for A $\$ 25.95$ for $30 \times 450 \mathrm{mg}$ tablets (this equates to a 15-day supply, the cost for 1 month is $\$ 51.90$ ).

The transition parameters are listed in table 2. The model parameters have been listed in table 3. Appendix 1 details the calculation of transition probabilities for the model.

\section{Probabilistic sensitivity analysis}

Uncertainty is addressed in the model using probabilistic sensitivity analysis. Statistical distributions were assigned to key model parameters to examine second-order

Table 2 Transition parameters used in the decision model

\begin{tabular}{llllll}
\hline Transition matrix & NYHA I & NYHA II & NYHA III & NYHA IV & Distribution \\
\hline NYHA I & 0.977 & 0.019 & 0.004 & 0.000 & Dirichlet \\
NYHA II & 0.008 & 0.981 & 0.010 & 0.001 & Dirichlet \\
NYHA III & 0.000 & 0.034 & 0.960 & 0.006 & Dirichlet \\
NYHA IV & 0.000 & 0.000 & 0.055 & 0.945 & Dirichlet \\
\hline
\end{tabular}

NYHA, New York Heart Association. 
Table 3 Parameters used in the decision model

\begin{tabular}{|c|c|c|c|c|}
\hline \multicolumn{5}{|l|}{ Probabilistic parameters } \\
\hline Parameter description & $\begin{array}{l}\text { Baseline } \\
\text { estimate }\end{array}$ & $\begin{array}{l}\text { Variation/ } \\
\text { SE (SD) }\end{array}$ & Distribution & Reference \\
\hline \multicolumn{5}{|l|}{ Hospitalisation } \\
\hline Length of stay in hospital estimate & 4.9 days & $\begin{array}{l}\alpha 0.1 \\
\beta 316.81\end{array}$ & $\gamma$ & 33 \\
\hline $\begin{array}{l}\text { Relative risk of hospitalisation with hawthorn extract } \\
\text { Mortality }\end{array}$ & 1.03651200 & 0.080800494 & Lognormal & 36 \\
\hline Excess mortality & & & $\beta$ & 28 \\
\hline $\begin{array}{l}\text { Probability of excess mortality given hospitalisation } \\
\text { class II }\end{array}$ & 0.01087776 & $\alpha 0.35916667$ & $\beta 2.55750000$ & \\
\hline $\begin{array}{l}\text { Probability of excess mortality given no } \\
\text { hospitalisation class II }\end{array}$ & 0.002620782 & $\alpha 0.43166667$ & $\beta 13.485000$ & \\
\hline $\begin{array}{l}\text { Probability of excess mortality given hospitalisation } \\
\text { class III }\end{array}$ & 0.01791369 & $\alpha 0.79666667$ & в 3.28666667 & \\
\hline $\begin{array}{l}\text { Probability of excess mortality given no } \\
\text { hospitalisation class III }\end{array}$ & 0.00674466 & $\alpha 0.72833333$ & $\beta 8.60500000$ & \\
\hline $\begin{array}{l}\text { Probability of excess mortality given hospitalisation } \\
\text { class IV }\end{array}$ & 0.05333974 & $\alpha 0.96416667$ & $\beta 1.03583333$ & \\
\hline $\begin{array}{l}\text { Probability of excess mortality given no } \\
\text { hospitalisation class IV }\end{array}$ & 0.00719464 & $\alpha 0.16583333$ & $\beta 1.83416667$ & \\
\hline Relative risk of mortality with hawthorn extract & 0.90336300 & 0.09507420 & Lognormal & 36 \\
\hline Utility & & & $\beta$ & 18 \\
\hline Utility of NYHA class I no hospitalisation & 0.815 & $\alpha 395.88$ & $\beta 89.86$ & \\
\hline Utility of NYHA class II no hospitalisation & 0.72 & $\alpha 661.95$ & в 257.42 & \\
\hline Utility of NYHA class III no hospitalisation & 0.59 & $\alpha 359.8075$ & $\beta 250.0357$ & \\
\hline Utility of NYHA class IV no hospitalisation & 0.508 & $\alpha 51.77$ & $\beta 50.1394$ & \\
\hline \multicolumn{5}{|l|}{ Fixed parameters } \\
\hline \multicolumn{5}{|l|}{ Hospitalisation } \\
\hline Probability for hospitalisation & 0.01518800 & 0.02397800 & 0.02397800 & 0.15397000 \\
\hline Probability no hospitalisation & 0.98481200 & 0.97602200 & 0.97602200 & 0.84603000 \\
\hline Costs & & & & 343537 \\
\hline Cost of hospitalisation & $\$ 2957.08$ & $\$ 4435.63$ & $\$ 4435.63$ & $\$ 5914.17$ \\
\hline Total cost for each NYHA class with hospitalisation & $\$ 3141.60$ & $\$ 4639.95$ & $\$ 4684.53$ & $\$ 6176.17$ \\
\hline Cost of each class with no hospitalisation & $\$ 130.30$ & $\$ 150.11$ & $\$ 194.69$ & $\$ 207.79$ \\
\hline Mortality & & & & \\
\hline Standardised death rate & 6.0 per 1000 & 6.0 per 1000 & 6.0 per 1000 & 6.0 per 1000 \\
\hline
\end{tabular}

uncertainty in the estimation of the parameter. Uncertainty was propagated through the model using Monte Carlo simulation, drawing parameter values at random 1000 times from the particular distributions. This generates a joint density of cost and QALY outcomes that summarises uncertainty in all model parameters.

\section{Net monetary benefit}

The incremental net monetary benefit (NMB) was calculated. The difference between the average net benefit of the standard treatment and the average net benefit of the standard treatment with hawthorn as an adjunct is equal to the incremental net benefit. The net benefit for each treatment is the increase in effectiveness multiplied by the amount the decision maker is willing to pay per QALY (\$40 000), minus the increase in cost.
The expected value of perfect information/expected value of partial perfect information

The results of the modelling will indicate whether, based on the currently available information, the new treatment should be recommended. This decision is always associated with a level of uncertainty, which raises the question of whether it is appropriate to conduct further research to better examine the potential value of the new treatment, and whether we can identify where this research needs to be directed. EVPI and expected value of partial perfect information (EVPPI) analysis have been used to address these questions.

EVPI analysis is a combination of the cost of making the wrong decision in terms of forgone health benefit and wasted resources, and the probability of making a wrong decision. This equates to the expected cost of uncertainty. With all uncertainty removed there would 
be economic savings from making the best decision and EVPI is a monetary value of these savings. EVPI provides an upper bound for spending on further research that reduces uncertainty in the decision. EVPPI follows the same principles, but examines individual parameters. ${ }^{39}$

For the model it has been assumed that the life of technology is 10 years and the number of eligible patients per annum has been estimated at 30000 . This estimate is derived from the estimate of 30000 new cases of chronic heart failure per annum.

\section{RESULTS}

For the standard treatment and standard treatment with hawthorn extract as an adjunct the total cost per patient was $\$ 4887.82$ and $\$ 6754.59$ QALYs were 2.40 and 2.42, respectively. This was an incremental cost of $\$ 1866.78$ and 0.02 QALYs, and the incremental cost-effectiveness ratio was $\$ 85160.33$ per QALY. A cost-effectiveness plane shows the joint density of cost and QALY outcomes from the Monte Carlo simulations (see figure 2). In figure 2, point $\mathrm{A}$ is the Incremental Cost Effectiveness Ratio (ICER). The variation in the model parameters can be seen in a series of histograms for each of the probabilistic parameters (see appendix 2).

\section{Cost-effectiveness acceptability curve}

Figure 3 shows the uncertainty around this estimate as a cost-effectiveness acceptability curve. At a willingness to pay a threshold of $\$ 40000$, the treatment with hawthorn extract has a 0.29 probability of being cost-effective. The probability of being cost-effective rises as the willingness to pay the threshold rises, for a threshold between $\$ 500000$ and $\$ 1000000$ the probability is 0.48 .

\section{Net monetary benefit}

For a threshold of $\$ 40000$, the average incremental NMB is $-\$ 1791.64$, the average NMB for the standard treatment is $\$ 92067.49$, and for the standard treatment with hawthorn as an adjunct $\$ 90$ 275.84. The treatment with hawthorn extract has a negative incremental net benefit, and would not offer good value for money for a decision maker.

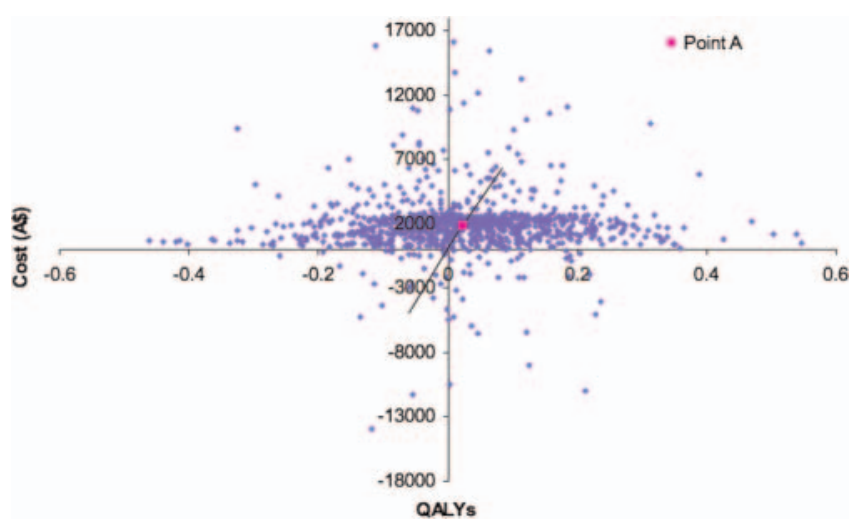

Figure 2 Cost-effectiveness plane. QALYs, quality-adjusted life years.

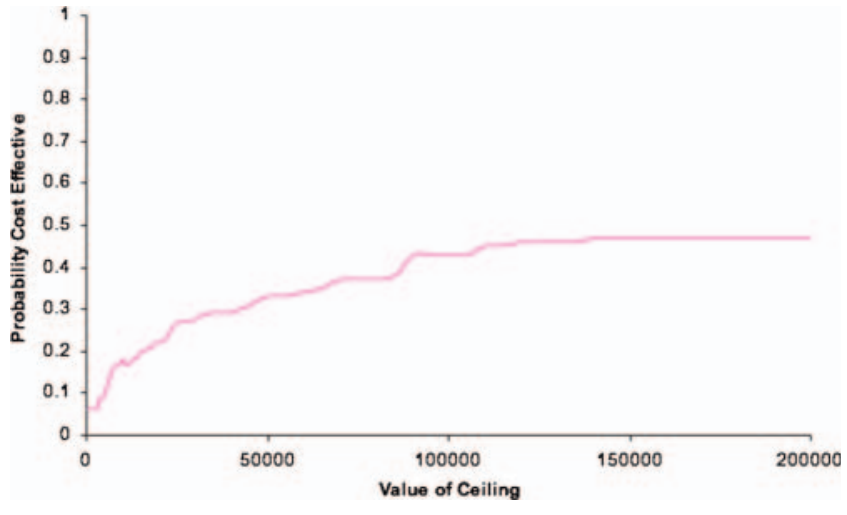

Figure 3 Cost effectiveness acceptability curve.

\section{Expected value of perfect information}

The population EVPI has been plotted in figure 4 for a cost-effectiveness threshold between $\$ 0$ and \$200000 per QALY. The threshold was continued in the analysis up to a threshold of $\$ 500000$ per QALY; however, this did not alter the slope of the curve, so the results up to \$200 000 have been shown.

If the population EVPI represented in figure 4 exceeds the expected costs of additional research, then it is potentially cost-effective to conduct further research.

At a threshold of $\$ 40000$, additional research is potentially cost-effective if research is not proposed to cost more than $\$ 325$ million.

If we proposed that additional research would cost $\$ 100$ million, it can be seen from figure 4 that this research would be potentially cost-effective at a threshold of just under $\$ 16000$. Even at a threshold of $\$ 0$ per QALY research would potentially be cost-effective as long as the cost of research did not exceed $\$ 15$ million.

The EVPI has indicated that further research is potentially cost-effective. The EVPPI was examined to establish where further research would be of most benefit.

\section{The expected value of partial perfect information}

The EVPPI was examined for six parameters/groups of parameters: transitions, average length of stay, excess

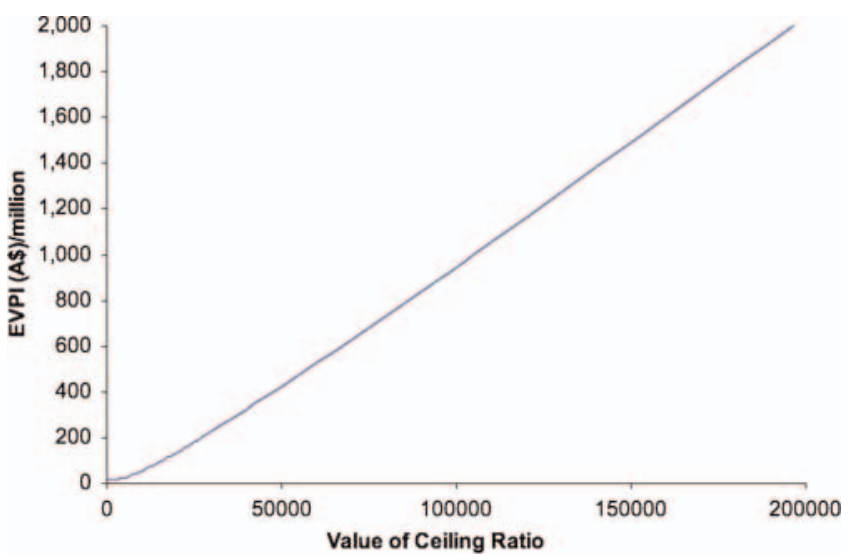

Figure 4 Expected value of perfect information (EVPI). 
mortality (cardiovascular mortality), relative risk of hawthorn, utilities and the relative risk of hospitalisation.

The results of the EVPPI analysis can be seen in figure 5 (and table 4). From both the table and figure it can be seen that all parameters and parameter groups have significant EVPPI, but the impact varies. Utilities (\$439 471050.98 ) has the highest EVPPI, and is therefore the most important target parameter/parameter group for further research.

\section{DISCUSSION}

In this modelling study, we examined the costeffectiveness of hawthorn extract in addition to standard treatment for heart failure in Australia. This treatment is not considered cost-effective given the current evidence. This is the first known attempt to examine the costeffectiveness of hawthorn extract in addition to standard pharmacological treatment of chronic heart failure in Australia. Economic evaluation has been conducted examining hawthorn extract and standard heart failure treatment in Germany and this research indicates that hawthorn extract was cost-effective in the study context; however, these studies were not considered rigorous enough for the data to be used in this study. ${ }^{10} 40$

EVPI analysis indicated that further research was likely to be of benefit, and EVPPI analysis indicated that research ideally should be targeted toward utilities. The potential costs of further research and the particular type or types that may be required are of crucial importance to the final decision. Further research to examine utilities will likely rely on primary data from randomised controlled trials such as the Eplerenone Post-acute Myocardial Infarction Heart Failure Efficacy and Survival Study (EPHESUS) and the SENIORS. ${ }^{18} 41$ Alternatively, such research would require the initiation of novel research with utilities as a main outcome. This is costly research and would certainly need to be estimated before being undertaken.

The models in any area of health vary in terms of the Markov states chosen, for example, when representing the severity of heart failure, hospitalisations and NYHA

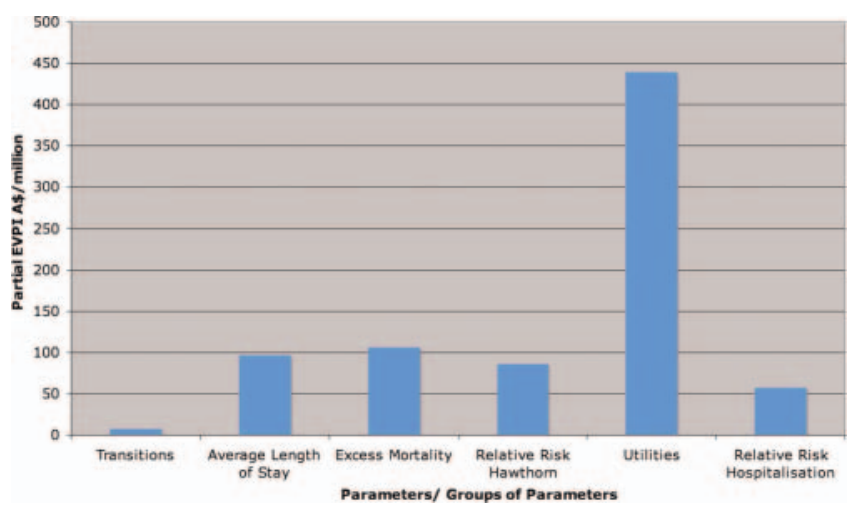

Figure 5 Expected value of partial perfect information (EVPPI).

\begin{tabular}{ll}
$\begin{array}{l}\text { Table } 4 \\
\text { groups }\end{array}$ & Partial EVPI values for parameters/parameter \\
\hline Parameters & Partial EVPI \\
\hline Transitions & $\$ 7153571.92$ \\
Average length of stay & $\$ 96900062.41$ \\
Excess mortality & $\$ 105833952.26$ \\
Relative risk hawthorn & $\$ 86323972.20$ \\
Utilities & $\$ 439471050.98$ \\
Relative risk hospitalisation & $\$ 56991399.70$ \\
\hline EVPI, expected value of perfect information.
\end{tabular}

classes of heart failure are both utilised. It is difficult to summarise the multitude of evidence and compare models as different model structures and methods are used, which potentially leads to different outcomes. ${ }^{42}$

The literature searches conducted for this study were comprehensive, although not to the standard of a systematic review. It is also often seen that the keywords chosen for CAM studies are not always uniform. The combination of these two factors may mean we have missed some of the research available. Our search was not reliant on databases alone, much of our information came from personal correspondence and a thorough search of reference lists, minimising the impact of the above limitations.

A limitation of this study was the relatively sparse data available for the Australian context. There are scarce data on the incidence and prevalence of heart failure. Estimates rely on information from a small number of large-scale population studies conducted in the USA and Europe. ${ }^{1}$ The study of mortality in Australia is complex, heart failure is considered a 'mode of death' not a 'cause of death'. Studies examining mortality in terms of the underlying cause of death, risk underestimating mortality in conditions such as heart failure. Mortality statistics are complicated by multiple comorbidities, which make the underlying cause of death difficult to identify. Lack of consensus about the diagnosis of heart failure also complicates recording of the cause of death, indeed complicating any examination of heart failure. It is difficult to isolate costs for heart failure. Heart failure is grouped by the Australian Institute of Health and Welfare as an 'other cardiovascular disease'. The exact contribution of heart failure to the burden of cardiovascular disease is at best an estimate.

Another limitation was the availability of evidence of the effectiveness of hawthorn extract. There are numerous studies supporting its use; however, very few studies examine final outcomes such as hospitalisation and mortality. ${ }^{12} 3032$ Previously conducted studies focus on reported outcomes including maximal workload, exercise tolerance, pressure-heart rate product, 6 min walk test and left-ventricular ejection fraction. There are suggestions in the literature that the use of hawthorn extract can actually decrease the use of standard pharmacological therapy and 
alter the progression of heart failure, but little rigorous evidence to support this. ${ }^{10}$ If the use of standard pharmaceuticals was decreased, and/or disease progression was altered and patients improved their NYHA class to a greater extent or remained in the less symptomatic classes for longer this would decrease costs and potentially change the costeffectiveness in favour of adding hawthorn extract as an adjunct to standard pharmacological treatment.

Should further evidence become available, the model can easily be updated and the results re-examined.

\section{CONCLUSION}

Our analysis indicates that based on currently available evidence, hawthorn extract is not cost-effective in addition to standard pharmacological treatment for chronic heart failure in Australia. EVPI and EVPPI analysis indicates that further research is warranted, particularly in the area of utilities, pending an assessment of the estimated costs of such research.

\section{Author affiliations}

${ }^{1}$ School of Population Health, University of Queensland, Brisbane, Queensland, Australia

${ }^{2}$ Faculty of Nursing, Midwifery and Health, University of Technology, Sydney, New South Wales, Australia

${ }^{3}$ School of Social Science, University of Queensland, Brisbane, Queensland, Australia

${ }^{4}$ School of Public Health and Institute for Health \& Biomedical Innovation, Queensland University of Technology, Brisbane, Queensland, Australia

Contributors EF, NG and JA were responsible for the conception and design of the research. EF carried out the data collection and economic analysis. EF was responsible for the original draft. All authors contributed equally to all other aspects including drafting and revising, and approved the final manuscript.

Funding This research received no specific grant from any funding agency in the public, commercial or not-for-profit sectors.

Competing interests None.

Provenance and peer review Not commissioned; externally peer reviewed.

Data sharing statement All requests for unpublished data should be directed to the corresponding author, Emily Ford, emily.ford1@uqconnect.edu.au

\section{REFERENCES}

1. Krum H, Stewart S. Chronic heart failure: time to recognise this major public health problem. Med J Aust 2006;184:147-8.

2. McLean AS, Eslick GD, Coats AJS. The epidemiology of heart failure in Australia. Int $J$ Cardiol 2007;118:370-4.

3. Agvall B, Borgquist L, Foldevi M, et al. Cost of heart failure in Swedish primary healthcare. Scand J Prim Health 2005;23:227-32.

4. Australian Institute of Health and Welfare. Heart failure: what of the future? Cat. No. AUS 34. Canberra: AlHW, 2003.

5. National Heart Foundation of Australia and the Cardiac Society of Australia and New Zealand (Chronic Heart Failure Guidelines Expert Writing Panel). Guidelines for the prevention, detection and management of chronic heart failure in Australia, 2006, 2006.

6. Krum H, Jelinek MV, Stewart S, et al. Guidelines for the prevention, detection and management of people with chronic heart failure in Australia in 2006. Med J Aust 2006;185:549-56.

7. Kaye DM, Krum H. Drug discovery for heart failure: a new era or the end of the pipeline? Nat Rev Drug Discov 2007;6:127-39.

8. Quinn B. Pharmacological treatment of heart failure. Crit Care Nurs Q 2007;30:299-306.
9. Furey A, Tassell M. Towards a scientific approach in the assessment of efficacy of an herbal preparation: hawthorn (Crataegus spp.). Eur $J$ Heart Fail 2008;10:1153-7.

10. Habs M. Prospective, comparative cohort studies and their contribution to the benefit assessments of therapeutic options: heart failure treatment with and without hawthorn special extract WS 1442 Forsch Komp Klas Nat 2004;11:36-9.

11. Pittler $\mathrm{MH}$, Schmidt K, Ernst E. Hawthorn extract for treating chronic heart failure: meta-analysis of randomised trials. Am J Med 2003;114:665-74

12. Guo R, Pittler MH, Ernst E. Hawthorn extract for treating chronic heart failure (Review). Cochrane Database Syst Rev 2009; (1). Art No: CD005312. doi: 10.1002/14651858.CD005312.pub2.

13. Griffin S, Claxton K, Sculpher M. Decision analysis for resource allocation in health care. J Health Serv Res Po 2008;13:23-30.

14. Kymes S. An introduction to decision analysis in the economic evaluation of the prevention and treatment of vision-related diseases. Ophthal Epidemiol 2008;15:76-83.

15. Buxton MJ, Drummond MF, Van Hout BA, et al. Modelling in economic evaluation: an unavoidable fact of life. Health Econ 1997;6:217-27.

16. Spencer M, Briggs A, Grossman R, et al. Development of an economic model to assess the cost effectiveness of treatment interventions for chronic obstructive pulmonary disease. Pharmacoeconomics 2005;23:619-37.

17. Rich MW, Nease RF. Cost-effectiveness analysis in clinical practice: the case of heart failure. Arch Intern Med 1999;159:1690-700.

18. Yao G, Freemantle N, Flather M, et al. Long-term cost-effectiveness analysis of nebivolol compared with standard care in elderly patients with heart failure: an individual patient-based simulation model. Pharmacoeconomics 2008;26:879-89.

19. Miller G, Randolph S, Forkner E, et al. Long-term cost-effectiveness of disease management in systolic heart failure. Med Decis Making Online First 2009.

20. Yao G, Freemantle N, Calvert M, et al. The long-term cost-effectiveness of cardiac resynchronization therapy with or without an implantable cardioverter-defibrillator. Eur Heart $J$ 2007;28:42-51.

21. Canter P, Thompson-Coon J, Ernst E. Cost effectiveness of complementary treatments in the United Kingdom: a systematic review. Br Med J 2005;331:880-1.

22. Dalziel K, Segal L, Mortimer D. Review of Australian health economic evaluation-245 interventions: what can we say about cost effectiveness? Cost Eff Resour Alloc 2008;6:9.

23. Thompson T, Feder G. Complementary therapies and the NHS uncertain evidence of cost effectiveness should not exclude complementary medicine from reviews and guidelines. $\mathrm{Br} \mathrm{Med} \mathrm{J}$ 2005;331:856-7.

24. Herman $P$, Craig B, Caspi O. Is complementary and alternative medicine (CAM) cost-effective? A systematic review. BMC Complem Altern M 2005;5:11.

25. Hollinghurst S, Sharp D, Ballard K, et al. Randomised controlled tria of Alexander technique lessons, exercise, and massage (ATEAM) for chronic and recurrent back pain: economic evaluation. $\mathrm{Br}$ Med $\mathrm{J}$ Online First 2008;337:a2656.

26. Kim N, Yang B, Lee T, et al. An economic analysis of usual care and acupuncture collaborative treatment on chronic low back pain: a Markov model decision analysis. BMC Complem Altern M 2010;10:274.

27. Flather M, Shibata M, Coats A, et al. Randomised trial to determine the effect of nebivolol on mortality and cardiovascular hospital admission in elederly patients with heart failure (SENIORS). Eur Heart J 2005;26:215-25.

28. Muntwyler J, Abetel G, Gruner C, et al. One-year mortality among unselected outpatients with heart failure. Eur Heart J 2002;23:1861-6.

29. Holubarsch CJF, Colucci WS, Meinertz T, et al. Survival and prognosis: investigation of crataegus extract WS 1442 in congestive heart failure (SPICE)-rationale, study design and study protocol. Eur J Heart Fail 2000;2:431-7.

30. Zick S, Gillespie B, Aaronson K. The effect of Crataegus oxycantha special extract WS 1442 on clinical progression in patients with mild to moderate symptoms of heart failure. Eur J Heart Fail 2008;10:587-93.

31. Zick S, Vautaw B, Gillespie B, et al. Hawthorn extract randomised blinded chronic heart failure (HERB-CHF) trial. Eur J Heart Fail 2009;11:990-9.

32. Cowper PA, DeLong ER, Whellan DJ, et al Economic effects of beta-blocker therapy in patients with heart failure. Am J Med 2004;116:104-11. 
33. Victorian Department of Health. Victorian health services policy and funding guidelines 2010-11 technical guidelines. Melbourne: Victorian Department of Health, 2010.

34. Queensland Health. Casemix funding model 2008-2009: component prices summary. Brisbane: Queenland Government, 2008.

35. Australian Government Department of Health and Ageing. Medicare Benefits Schedule (MBS) online. Canberra: Australian Government Department of Health and Ageing, 2010.

36. Holubarsch CJF, Colucci WS, Meinertz T, et al. The efficacy and safety of crataegus extract WS 1442 in patients with heart failure: the SPICE trial. Eur J Heart Fail 2008;10:1255-63.

37. Australian Government Department of Health and Ageing. Schedule of pharmaceutical benefits. Canberra: Australian Government Department of Health and Ageing, 2010.
38. Australian Bureau of Statistics. 3302.0-Deaths, Australia, 2008.

39. Drummond MF, Sculpher MJ, Torrance GW, et al. Methods for the economic evaluation of health care programmes. 3rd edn. Oxford: Oxford University Press, 2007.

40. Koller M, Lorenz W, Aubke W, et al. Crataegus special extract WS 1442 in the treatment of early stages of CHD-associated heart failure. MMW Fortschr Med 2006;148:42.

41. Gohler A, Geisler BP, Manne J, et al. Utility estimates for decision-analytic modelling in chronic heart failure: health states based on New York heart association classes and number of rehospitalisations. Value Health 2009;12:185-7.

42. Turner D, Raftery J, Cooper K, et al. The CHD challenge: comparing four cost-effectiveness models. Value Health 2011; 14:53-60. 